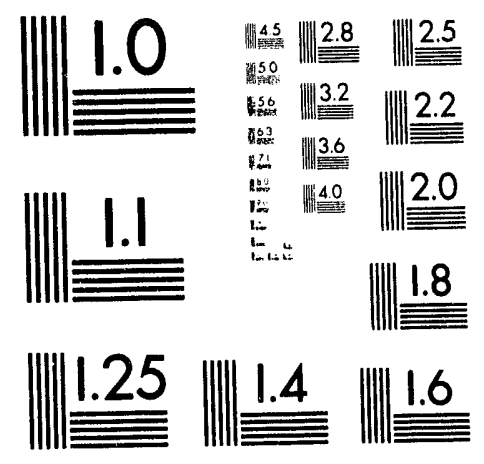



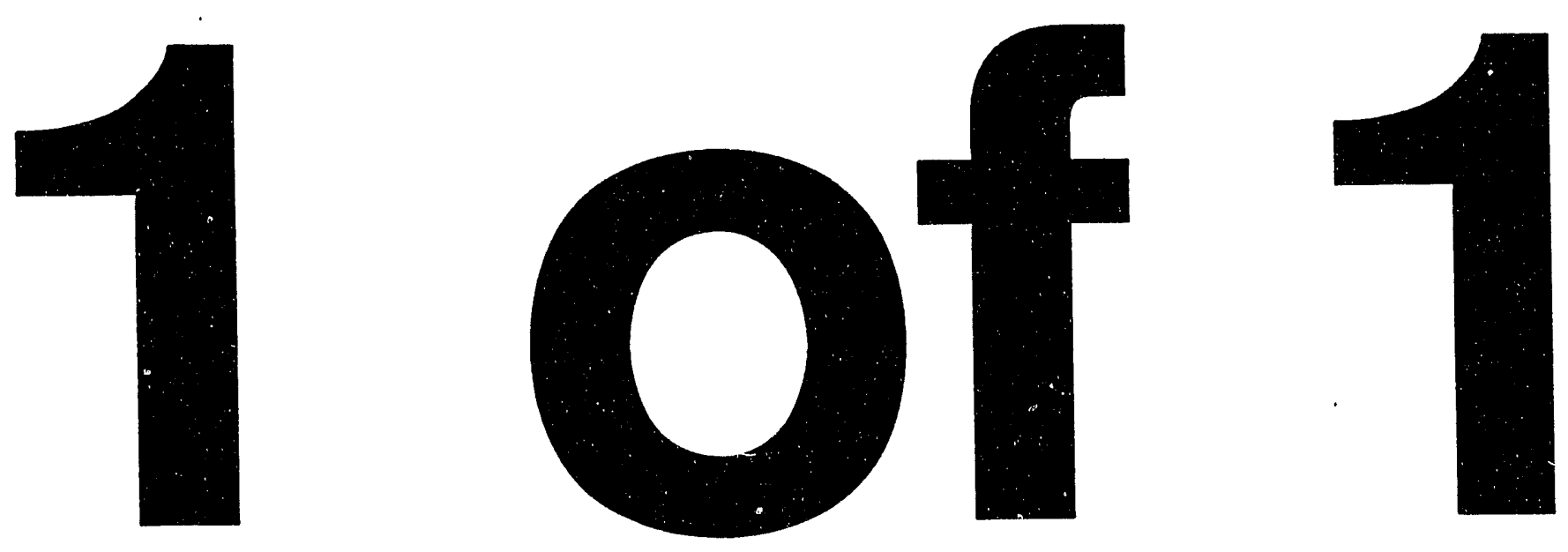


\section{POINTING CONTROL DESIGN \\ FOR \\ AUTONOMOUS SPACE VEHICLE APPLICATIONS}

\section{K. D. YOUNG}

This paper was prepared for presentation at

the 12th World Congress of the International Federation of Automatic Control,

July 19-23 1993, Sydney, Australia

March 1993

This work was performed under the auspices of the U.S. Department of Energy by Lawrence Livermore National Laboratory under contract No. W-7405-Eng-48. 


\title{
POINTING CONTROL DESIGN FOR AUTONOMOUS SPACE VEHICLE APPLICATIONS
}

\author{
K. D. YOUNG* \\ - Laurence Livenmore National Laboralory, Universily of Califomia, Livermore, California 94550 U.S.A.
}

\begin{abstract}
This paper addresses the design of pointing control systems for autonomous apec vehicles. The function of the pointing control aystem in to keep distant orbiting objects within the field-of-view of an on-board optical sensor. We outline the development of novel nonlinear control algorithms which exploit the suilability of on-board sensors. Simulation results comparing the performance of the different pointing control implementations are presented.
\end{abstract}

Key Words: Attitude Pointing Control; Nonlinear Control; Variable Structure Control

\section{INTRODUCTION}

Design of space vehicle attitude control systems where large angular rotations are required has been addressed using the concept of Euler axis of rotation and quaternion feedback control (Moriensen (1986), Wie and Barba (1985), Vadali (1986), VanDenBosch et. al. (1986), Wie et. al.(1989)). Euler axis of rotation is preferred since it defines an axis about which if the specified scalar angle of rotation is performed provides the most direct transition between two orientations of the space vehicle. Rotational maneuvers which execute the Euler axis rotation are considered to be optimal maneuvers. The existing works dealt with rest-torest re-orientation manuevers where the vehicle body rates are regulated, and vehicle quaternion feedback is employed. Lyapunov synthesis methods are mostly applied for design and stability analysis, although variable strcuture control design method has also been considered.

In this paper, we examine the design of pointing control systems for autonomous space vehicle applications where wide Field-Of-View (FOV) on-boand optic sensors are available to track distant orbiting objects, and the primary attitude control actuators are in the form of thrusters. The design objective of the pointing control loop is to align the Line-ofSight (LOS) of the orbiting object to the center of the imaging plane of the optic sensor. For autonomous operations, the pointing control system utilizes, in addition to the optic sensor measurements, the vehicle's angular rates which are measured by means of gyros. As the orbiting object moves in space relative to the space vehicle, the pointing control system is to be designed such that the LOS alignment is maintained.

\section{VEHICLE KINEMATICS AND DYNAMICS}

The oriexication of the orbiting vehicle is defined with respect to a Cartesian coordinate frame whose origin is at the center of Earth (Earth Centered Inertial (ECI) frame). The difference in orientation between the Body Fixed (BF) coordinate and the ECI frame is expressed by its Euler parameters, or quaternions which consist of a scalar part $\eta_{V E}$, and a three component vector part ovE. Angular rates of rotation with respect to the three orthogonal BF frame axes characterize the vehicle's angular motion dymamics. Let $w$

be $\mathrm{a} 3 \times 1$ vector whose first component is the roll rate, the second is pitch, and the third is yaw rate. The quaternions evolve in time according to ${ }^{1}$ :

$$
\begin{aligned}
& \dot{q}_{V E}=-\frac{1}{2}\left(\omega^{\times} q_{V E}-\omega \eta_{V E}\right), \\
& \dot{\eta}_{V E}=-\frac{1}{2} \omega^{T} q_{V E} .
\end{aligned}
$$

The body rates satisfy rigid body rotational dynamics equation:

$$
J \dot{\omega}=-\omega^{\times} J \omega+\tau,
$$

where $\tau$ denotes a three component vector whose elements represent torque applied to the vehicle with respect to the body axes, and $J$ is the moment of inertia matrix.

\section{ON-BOARD IMAGING SENSOR}

An optical sensor is rigidly mounted to the vehicle body. Gimballed mounts are eliminated by other vehicle system engineering design constraints. It is assumed that the pointing axis of the sensor coincides with vehicle's $X$ (roll) axis. The imaging plane of the optics is in the vehicle's $Y$ (pitch)$\mathrm{Z}$ (yow) plane. A distant orbiting object, if detected within the field-of-view of the sensor, is registered on the imaging plane and decoded as a pair of coordinates which respresent the "centroid" of the object image. Equivalently, its location on the plane can be characterized by an azimuth angle $\theta_{A}$, and an elevation angle $\theta_{E}$. Given these angles, an unit line-of-sight (LOS) vector is defined:

$$
\begin{aligned}
& \vec{p}=r_{P}^{-1}\left(1, \tan \theta_{A}, \tan \theta_{E}\right), \\
& r_{p}=\left(1+\tan ^{2} \theta_{A}+\tan ^{2} \theta_{E}\right)^{\frac{1}{2}} .
\end{aligned}
$$

Three directional cosine angles, $\alpha, \beta, \gamma$, are derivable from $\vec{p}:$

$$
\cos \gamma=1 / r_{p}, \cos \beta=\tan \theta_{A} / r_{p}, \cos \alpha=\tan \theta_{E} / r_{p}(6)
$$

$$
\begin{aligned}
& { }^{1} \vec{a}=\left(a_{1}, a_{2}, a_{3}\right) \text { is expresed as } 3 \times 1 \text { vector } a^{T}=\left[a_{1}, a_{2}, a_{3}\right], a^{\times} \triangleq \\
& {\left[\begin{array}{ccc}
0 & -a_{3} & a_{2} \\
a_{3} & 0 & -a_{1} \\
-a_{2} & a_{1} & 0
\end{array}\right]}
\end{aligned}
$$




\section{POINTING CONTROL}

The pointing control algorithms we developed are based on Euler rotation. Fror' ' issical mechanics (Goldstein(1981)), it is known that given two coordinate systems with different orientations, there exists an axis of rotation (the so-called Euler axis of rotation) $\vec{\lambda}$ and an angle $\phi$ such that rotating one of the coordinate system about $\vec{\lambda}$ by $\phi$ produces two coordinate systems with identical orientations. For the orbiting vehicle, the goal of pointing the roll axis at the distant orbiting object is equivalent to aligning the roll axis with the LOS vector $p$. By adopting the LOS vector as the $\mathrm{X}$ axis of $\mathrm{a}$ fictituous rigid body and defining the other two orthogonal axes by right hand rule, a new coordinate system (the LOS frame) is created. The pointing control goal is translated into matching the orientations of the LOS and the BF frame. The Euler axis of rotation is defined with the directional cosine angles:

$$
\vec{\lambda}=\left(\begin{array}{lll}
0 & -\frac{\cos o}{\sin \gamma} \frac{\cos \rho}{\sin \gamma}
\end{array}\right),
$$

and the requisite angle of rotation is $\gamma$. Figure 1 shows the relative orientations of the LOS and BF frames. The quanternions that characterize the orientation of the $\mathrm{BF}$ frame relative to the LOS frame are:

$$
\overrightarrow{q V L}=(\sin \gamma / 2) \vec{\lambda}, \quad \eta_{V L}=\cos \gamma / 2 .
$$

The quaternions for the orientation of the LOS frame relative to the BF frame are:

$$
\vec{q}_{L V}=-\vec{q}_{V L}, \quad \eta_{L V}=\eta_{V L} .
$$

Using trigonometric identities, we express $\vec{q}_{L V}$ as a threc component vector in the form:

$$
q_{L V}^{T}=\left[\begin{array}{lll}
0 & \frac{\cos \alpha}{2 \cos \gamma / 2} & -\frac{\cos \rho}{2 \cos \gamma / 2}
\end{array}\right] .
$$

\subsection{A Lyapunov Design}

A pointing control law is derived below using a Lyapunov stability argument. It is composed of a feedforward term to compensate for cross axis rate couplings, and rate and orientation error feedback where the orientation error is expressed in terms of $q_{L V}$ :

$$
\begin{aligned}
& \tau=\omega^{\times} J \omega-D\left(\omega-\omega_{L}\right)-K q_{L v}, \\
& D=\operatorname{diag}\left(d_{1}, d_{2}, d_{3}\right), \quad K=\operatorname{diag}\left(k_{1}, k_{2}, k_{3}\right),
\end{aligned}
$$

where $\alpha_{i}>0, k_{i}>0, i=1,2,3$ and $\omega_{L}$ is a $3 \times 1$ angular rate vector whose components are the rates of rotation about the LOS frame's axes. It is assumed that the body rates of the LOS frame remain constant during the orbital encounter. The closed loop system stability is examined via a Lyapunov function candidate:

$$
\begin{gathered}
V=\frac{1}{2}\left(\omega-\omega_{L}\right)^{T} K^{-1} J\left(\omega-\omega_{L}\right)+ \\
+\left(q_{V E}-q_{L E}\right)^{T}\left(q_{V E}-q_{L E}\right)+\left(\eta_{V E}-\eta_{L E}\right)^{2},
\end{gathered}
$$

where $q_{L E}, \eta_{L E}$ are the quatemions of the LOS frame with respective to the ECI frame. Since $J$ is positive definite, and $k_{i}$ 's are chosen to be positive, $V$ is positive definite except at the origin where $\omega=\omega_{L}, q_{V E}=q_{L E}$, and $\eta_{V E}=$ $\eta_{L E}$. The Lyapunov stability proof hinges on an equivalent representation for $q_{L V}$ which is expressed in terms of the quaternions $q_{V E}$ and $q_{L E}$ :

$$
q_{V L}=-q_{L V}=\eta_{V E} q_{L E}-\eta_{L E} q_{V E}-q_{V E}^{x} q_{L E} .
$$

It has been proved (the proof is omitted due to page linits) that

$$
\dot{V}=-\left(\omega-\omega_{L}\right)^{T} K^{-1} D\left(\omega-\omega_{L}\right)<0 .
$$

Thus, in the closed loop system, the origin is an asymptotirally stable equilibrium, i.e., the angular rates of the orbiting vehicle synchronize with that of the LOS asymptotically, and the quaternions of the vehicle tend to the same as that of the LOS frame. This means that the pointing axis would eventually be in perfect alignment with the LOS.

\subsection{Feedback Control Realization}

The feedback mechanisms in the pointing control law become clear if the last term in Eqn.(12) are expressed more explicitly in terms of the azimuth and elevation angles. Denote the feedback control in Eqn.(12) as $\tau^{f}$,

$$
\begin{aligned}
\tau^{f}=-D\left(\omega-\omega_{L}\right) & -K q_{L V}=-\left[\begin{array}{l}
d_{1}\left(\omega_{1}-\omega_{L, 1}\right) \\
d_{2}\left(\omega_{2}-\omega_{L, 2}\right) \\
d_{3}\left(\omega_{3}-\omega_{L, 3}\right)
\end{array}\right]+ \\
& +\left[\begin{array}{c}
0 \\
-k_{2} \frac{r_{1}^{\frac{1}{2}}}{\sqrt{2}\left(1+r_{p}\right)^{\frac{1}{2}}} \tan \theta_{E} \\
+k_{3} \frac{r_{2}^{\frac{1}{2}}}{\sqrt{2}\left(1+r_{p}\right)^{\frac{1}{2}}} \tan \theta_{A}
\end{array}\right] .
\end{aligned}
$$

From these expressions, we observe that:

1. The roll torque (the first component of $\tau^{f}$ ) provides damping only. Orientation error in the roll axis is sutomatically nulled by servoing actions in the pitch and yow channels.

2. The servoing actions which operate on the pitch and yaw orientation errors are proportional to the tangents of the azimuth and elevation angles of the optical sensor.

For a vehicle whose body axes conicide with the principal axes, the inertia matrix $J$ is diagonal and its diagonal elements $J_{i}, i=1,2,3$ are the principal moments of inertia. The roll dynamics are decoupled from the pitch-yaw dynamics, and the closed loop system is reduced to ':

$$
\begin{aligned}
& J_{1} \dot{\omega}_{1}=-d_{1}\left(\omega_{1}-\omega_{L, 1}\right), \\
& J_{2} \dot{\omega}_{2}=-d_{2}\left(\omega_{2}-\omega_{L, 2}\right)-k_{2} r\left(\theta_{E}, \theta_{1}\right) \tan \theta_{E}, \\
& J_{3} \dot{\omega}_{3}=-d_{3}\left(\omega_{3}-\omega_{L, 3}\right)+k_{3} r\left(\theta_{E}, \theta_{A}\right) \tan \theta_{A} .
\end{aligned}
$$

where

$$
r\left(\theta_{E}, \theta_{A}\right) \triangleq\left(\frac{r_{p}}{2\left(1+r_{p}\right)}\right)^{\frac{1}{t}}
$$

is a norm-like measure of the angular deviations from boresighting the distant object.

From a feedback control realization viewpoint, it is advantageous to replace $\omega_{L}$ which is a vector expresod in the LOS frame by $\omega_{L}^{V}$ - 8 vector whose componenis are the rotations of the LOS frame as detected and compulad from the vehicle's on-board sensors. For the pilch and yaw axes, the azimuth and elevation angles can be used anoular displacement state variables. From the relative goumetry of the LOS and the body frame, we have

$$
\begin{aligned}
& \dot{\theta}_{E}=\omega_{2}+\dot{\sigma}_{E}, \\
& \dot{\theta}_{\boldsymbol{A}}=-\omega_{3}+\dot{\sigma}_{\boldsymbol{A}},
\end{aligned}
$$

where $\dot{\sigma}_{E}$ and $\dot{\sigma}_{A}$ are the rotations of the LOS frame $m$ th respect to the pitch and yaw axis respectively for the roll axis, the angular rotation of the object as $1 \mathrm{~mm}$ the imaging plane is the third displacement stake manable

$$
\dot{\theta}_{R}=\omega_{1}-\omega_{L, 1} \triangleq \omega_{R} .
$$

Note that the steady state value of $\theta_{R}$ define the onentation of the LOS frame in the plane normal to the L.OS. Defining the rate errors as

$$
\begin{aligned}
& \omega_{E} \triangleq \omega_{2}+\dot{\sigma}_{E}=\dot{\theta}_{E}, \\
& \omega_{A} \triangleq \omega_{3}-\dot{\sigma}_{A}=-\dot{\theta}_{A},
\end{aligned}
$$

'For vehicles with non-diegonul inertis matrix, this ampitication can be axcomplished by premultiplying the RHS of tian (12) by $J$ 
the resulting feeback control realization and the associated stae space model of the closed loop system are summarized below.

$$
\begin{aligned}
\tau^{\prime} & =-\left[\begin{array}{l}
d_{1} \omega_{R} \\
d_{2} \omega_{E} \\
d_{3} \omega_{A}
\end{array}\right]+\left[\begin{array}{c}
0 \\
-k_{2} r\left(\theta_{E}, \theta_{A}\right) \tan \theta_{E} \\
+k_{3} r\left(\theta_{E}, \theta_{A}\right) \tan \theta_{A}
\end{array}\right] \\
\dot{\theta}_{R} & =\omega_{R} \\
J_{1} \dot{\omega}_{R} & =-d_{1} \omega_{R}, \\
\dot{\theta}_{E} & =\omega_{E} \\
J_{2} \dot{\omega}_{E} & =-d_{2} \omega_{E}-k_{2} r\left(\theta_{E}, \theta_{A}\right) \tan \theta_{E}, \\
\dot{\theta}_{A} & =-\omega_{A} \\
J_{g} \dot{\omega}_{A} & =-d_{3} \omega_{A}+k_{3} r\left(\theta_{E}, \theta_{A}\right) \tan \theta_{A} .
\end{aligned}
$$

Since $\left.2^{-1 / 2}<r\left(\theta_{E}, \theta_{A}\right)\right)<2^{-1}$, the stability of the pitch and yaw subsystems can be attributed to its similarity to a coupled spring-mass system where nonlinear stiffness exists in the equivalent mechancial springs - spring forces become unbounded only at the singular points $\theta_{E}=\theta_{A}=\pi / 2$ which are physically unrealistic even in wide-field-of-view optics.

\subsection{Feedback Control Gain Design}

The design of the control gains $d_{i}$ 's and $k_{i}$ 's for the pitch and yow channels are simplified by using that the following approximations for the nonlinearities for azimuth and elevation angles in the range $0 \leq \theta_{A}, \theta_{E} \leq \pi / 4$ :

$$
\begin{aligned}
r\left(\theta_{E}, \theta_{A}\right) & \approx \frac{1}{2}, \\
\tan \theta_{A} & \approx \theta_{A}, \quad \tan \theta_{E} \approx \theta_{E} .
\end{aligned}
$$

The control gains can then be selected for a linear system whose closed loop characteristic equation is:

$$
J_{i} s^{2}+d_{i} s+\frac{k_{i}}{2}=0, \quad i=2,3
$$

For the roll channel, the time constant of the roll rate error decay is $J_{1} / d_{1}$. Standard linear control design methods are applicable to meet transient response and steady state performance criteria. For large initial off-boresight angular errors, implying wider field-of-view optics, there are two options for the design. First, the same gain values as calculated from the above equation can be used. For large initial errors, the effective damping is smaller than as predicted in the linear design. This is due to the large linearized position gain for large angles, whereas the velocity gain is constant. Thus, the linear design can be used as a conservative design even for large initial errors. Alternatively, a direct compensation of the nonlinearities is also feasible. For the particular feedback control realization using azimuth and elevation angles as orientation state variables, replacing the last term in Eqn.(26) by contant gain feedback of the respective offboresight angles reduces t'e design problem to essentially a linear one.

\section{CONTRC L ACTUATOR CONSTRAINTS}

The use of constant magnitude thrusters in pointing control for autonomous space vehicles requires a Pulse-WidthModulation (PWM) implementation of the developed control ilgorithms. This means that discrete value discontinuous control signals are devised to approximate the contin uous control signals. Alternatively, discontinuous control design methods such as variable strcuture control (VSC) can be directly applied to compute the feedback parameters. One of the major advantage of this approach is the superb disturbance rejection and robustness properties of VSC. This means that it is not necessary to provide a feedforward term in the pointing control law. The VSC pointing control design is summarized below. The control torques are computed as:

$$
\tau_{i}= \begin{cases}\tau_{i}^{+}, & \text {if } \sigma_{i}\left(\theta_{E}, \theta_{A}\right)>\epsilon_{i} \\ 0, & \text { if } \mid \sigma_{i}\left(\theta_{E}, \theta_{A}\right) \leq \epsilon_{i}, \quad i=2,3 . \\ \tau_{i}, & \text { if } \sigma_{i}\left(\theta_{E_{E}}, \theta_{A}\right)<-\epsilon_{i}\end{cases}
$$

where a deadzone is established in an $c_{1}$ neighborhood of the switching surfaxe $\sigma_{i}(\cdot, \cdot)=0$. A constant torque $\bar{F}_{i}$ is applied by the firing one of two thrusters which exert forces in opposite directions. The signs of the torques for the pitch and yow channels are:

$$
\begin{aligned}
& \tau_{2}^{+}=-\tau_{2}, \quad \tau_{2}=+\tau_{2}>0, \\
& \tau_{3}^{+}=\tau_{3}>0, \quad T_{3}=-\tau_{3},
\end{aligned}
$$

Design of the switching surfaces is reduced to choosing the parameters $c_{2}, c_{3}$. From the sliding mode condition, i.e., $\sigma_{2}=\sigma_{3}=0$, the ideal sliding mode dynamics govern the transient behavior of the azirnuth and elevation angles:

$$
\begin{aligned}
& \dot{\theta}_{E}=-\operatorname{cor}\left(\theta_{E}, \theta_{A}\right) \tan \theta_{E}, \\
& \dot{\theta}_{A}=-\operatorname{cor}\left(\theta_{E}, \theta_{A}\right) \tan \theta_{A} .
\end{aligned}
$$

Using the same appraximations as in Eqn.(34), the time constants for the arimuth and elevation channels are $-2 / c_{2}$, and $-2 / c 3$ repsectively. For initial off-boresight angles larger than $\pi / 4$, the effective decay rates are higher. Thus these time constants provide conservative estimates of the transient decaying time in sliding mode.

An alternative to the deadzone is to implement an PWM realization of a continuous linear feedback signal,

$$
\tau_{i}=\kappa_{i} \frac{\sigma_{i}\left(\theta_{E}, \sigma_{A}\right)}{\epsilon_{i}}, \quad \text { if }\left|\sigma_{i}\left(\theta_{E}, \theta_{A}\right)\right| \leq \epsilon_{i} \quad, \quad i=2,3,
$$

wher $\kappa_{2}=-1, \kappa_{3}=1$.

\section{SIMULATION STUDY}

For the purpose of checking the peformance of the various pointing control implmentations, we simulate the pointing and tracking performance of the pitch-yaw loops. For ease of reference, we normalize the torque input to the vehicle axis by the respective principal moment of ivertia such that the control inputs areangular accelerations. The dynamic performance of the pointing control loop depends on the pitch and yaw motion. Thus we focus on the pitch and yaw loop control design. For the control gain selection, we specify a worst case transient response time constant of $0.1 \sqrt{2}$ seconds. According to the characteristic equation and the desired double poles at $-5 \sqrt{2}$,

$$
\begin{aligned}
& d_{2}=d_{3}=10 \sqrt{2}, \\
& k_{3}=d_{3}=100,
\end{aligned}
$$

which correspond to a damping ratio of $\xi=1$.

Disturbance rejection properties of the pointing control loops are tested. with a sawtooth time profile to represent the effects of time-varying disturbance torques which change signs abruptly. The disturbance torque is similarly scaled, and therefore is measured in rad./sec. ${ }^{2}$. The magnitude of the disturbance is $\pm 10 \mathrm{rad} . / \mathrm{sec}^{2}$, and its period is 100 msec. This is a severe, persistingly exciting disturbance for the control authority assumed. The disturbance has zero mean. The same disturbance is applied to the aximuth and elevation channels simultaneously.

Initial off-boresight errors correspond to an sicinuth angle of $\theta_{A}(0)=1.5658$ radians, and an elevation angle of 0.1 radians are chosen for the simulation. The initial azimuth angle is deliberately chosen to be very close to the singularity of the tangent function in order to test the robustness of the linear control design. Figure 2 shows the time responses with the continuous time pointing control. The time response of the azimuth angle is divided by $0.1 \theta_{A}(0)$ to permit a comparison of the small and large angle responses. Whereas the elevation angle shows the predicted transient response, the overshoot caused by the reduced damping for large angle is exhibited in the scaled azimuth angle plot. The effects of the PWM implementation of the continuous time pointing control law are shown in Figure 3. The constant normalized torque level is set at $10 \mathrm{radians} / \mathrm{sec}^{2}$. A 
$100 \mathrm{~Hz}$ sampling rate $(T=10 \mathrm{~m} \mathrm{sec}$.) is chosen for the computation of the sampled pointing control law. For the PWM implementation, a minimum pulse on period of 1 msec. is assumed. Control input saturation in the azimuth channel slows the error decay rate, as well as reducing the overshoot. The VSC pointing control, whose responses are given in Figure 4, is implemented as a discrete time control law with a sampling period of $10 \mathrm{msec}$. The switching surface parameters $c_{2}$ and $c_{3}$ are chosen to yield a time constant of $0.2 \mathrm{sec}$. for small initial angular errors. This corresponds to:

$$
c_{2}=c_{3}=10 \text {. }
$$

In both of these figures, the overshoot characteristics of the continuous time control are present for large azimuth initial error. Control input saturation in the azimuth channel lengthens the settling time.

\section{CONCLUSIONS}

Design of pointing control systems for autonomous space vehicles which utilizes on-board optical sensors for the detection of other orbiting objects can be simplified by adopting a new state space model which compactly represents the essential pointing system dynamics. The res!'ting pointing control laws are connected to those control laws designed for lange angle maneuvers for spacecrafts via Euler axis rotations. The dynamic variables required for feedback however are directly measurable in the present design, as compared to requiring quaternion computations in the previous designs. Control gain selections in the present design are based on standart linear design methods where the transient performance paremeters, such as damping ratio and time constants, are directly translated in the gain parameter space. This is in contrast to the Lyapunov methods in previous designs using Euler axis of rotations where asymptotic stability is the primary design objective, and transient performance is indirectly controlled via the rate of convergence of the Lyzpunov function. Within the current design framework, we compared the PWM and the VSC implementation of the pointing control law, both of which utilize control pulses to point. We found that VSC is more robust than PWM to both cross couplings between the pitch and vaw channels, and external distcontinuous disturbances.

\section{REFERENCES}

Mortensen, R. (1968). A globally stable linear attitude regulator. International Joumal of Control, Vo. 8, No. 3, pp. 297-302, .

Wie, B. and P. M. Barbs (1985). Quatemion Feedback for Spacecraft Large Angle Maneuver. Journal of Guidance, Control and Dymamics, Vol. 8, No. 3, pp. 360365.

Vadali, S. R. (1986). Variable Structure Control of Spacecraft Large Angle Maneuver. Journal of Cuidance, Control and Dynamics, Vol. 9, No. 1, pp. 235-239.

vanDenBosch, P. P. J. et. al. (1986). Adaptive Attitude Control for Large Angle Slew Maneuvers. Automatica, Vol. 22, No. 2, pp. 209-215.

Wie B., H. Weiss, and A. Arapostathis (1989). A Quaternion Feedback Regulator for Spacecraft Eigenaxis Rotations. Journal of Guidance, Control and Dynamics, Vol. 12 , No. 3, pp. $375-380$.

Goldstein, G. (1981) Classical Mechanics, Second Edition, Addision-Wesley.

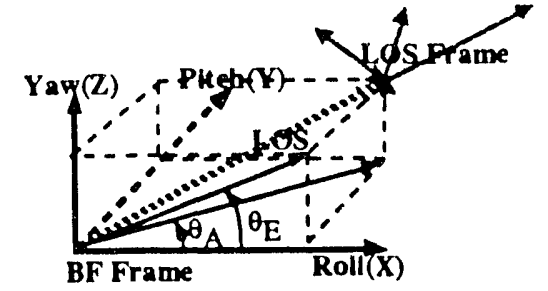

Figure 1: Body fixed reference frame and the LOS frame.

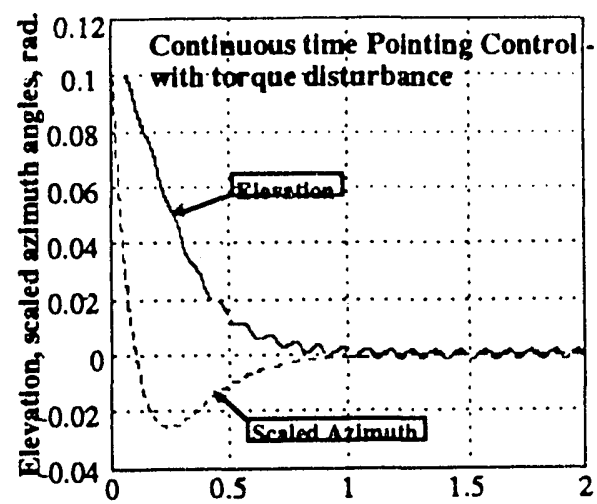

Figure 2: Elevation and azimuth angles: continuous time.

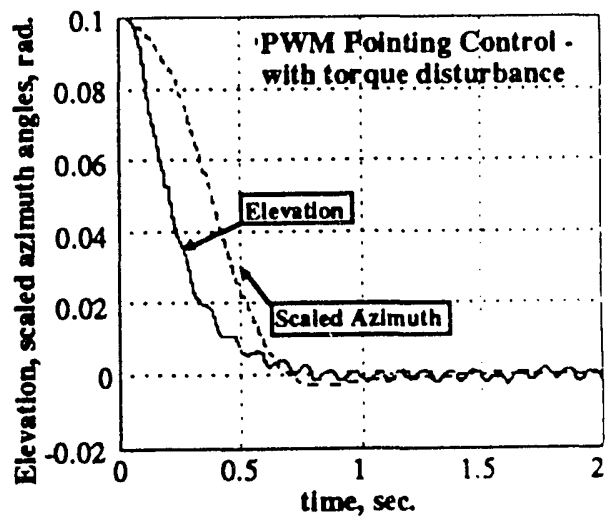

Figure 3: Elevation and azimuth angles: PW.M

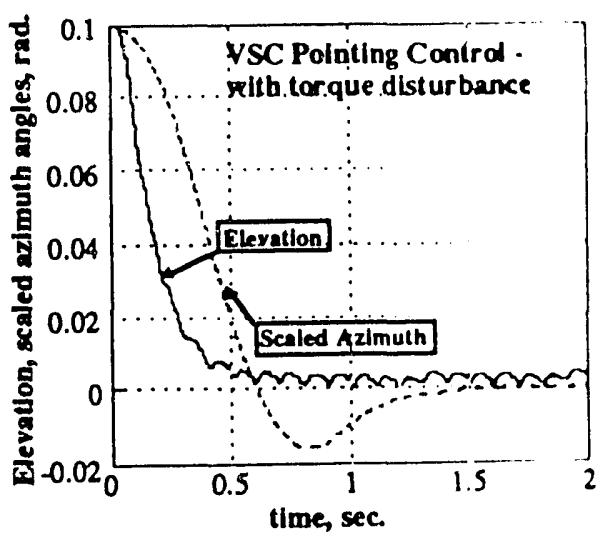

Figure 4: Elevation and azimuth angle: VSC 

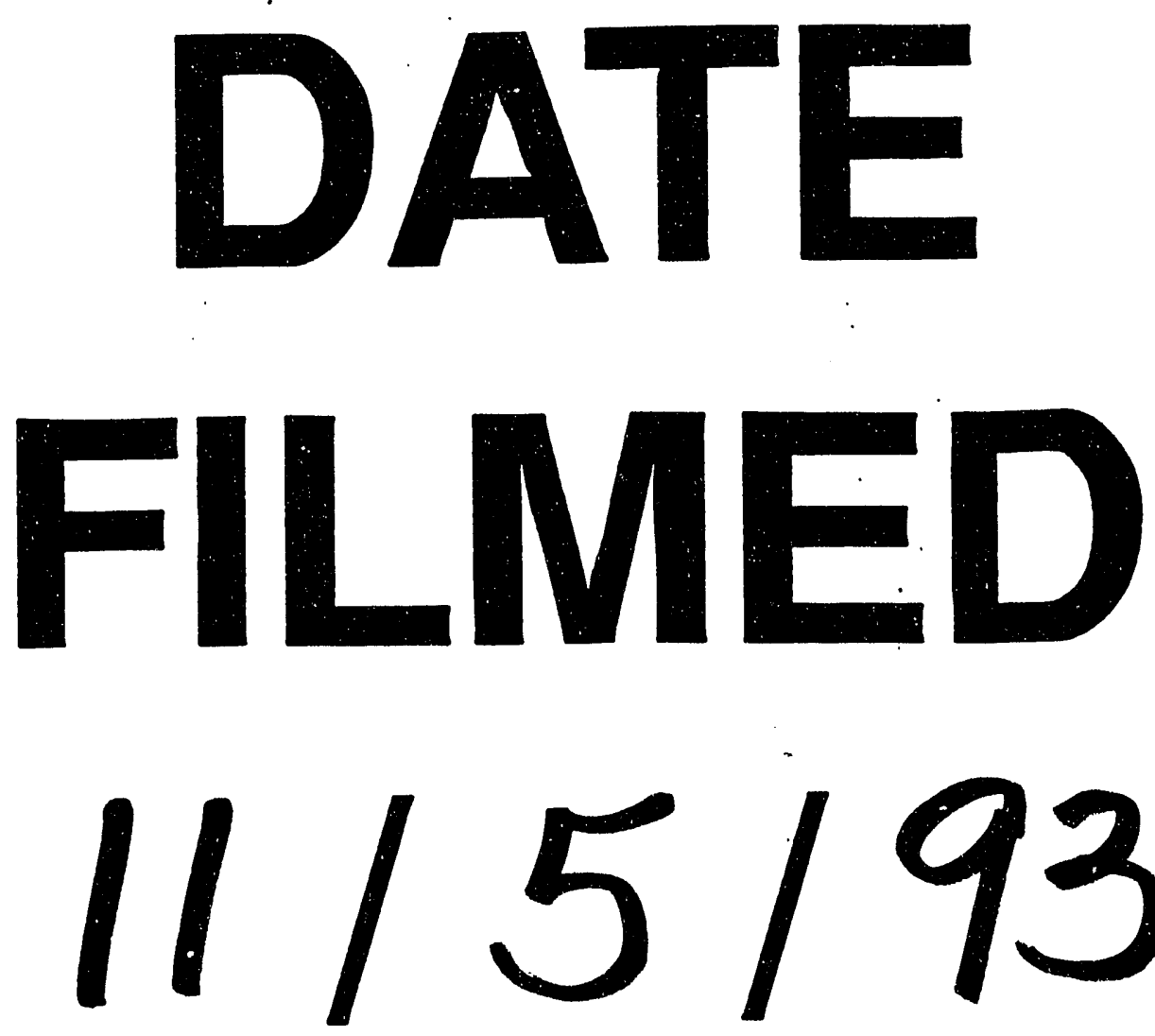

93
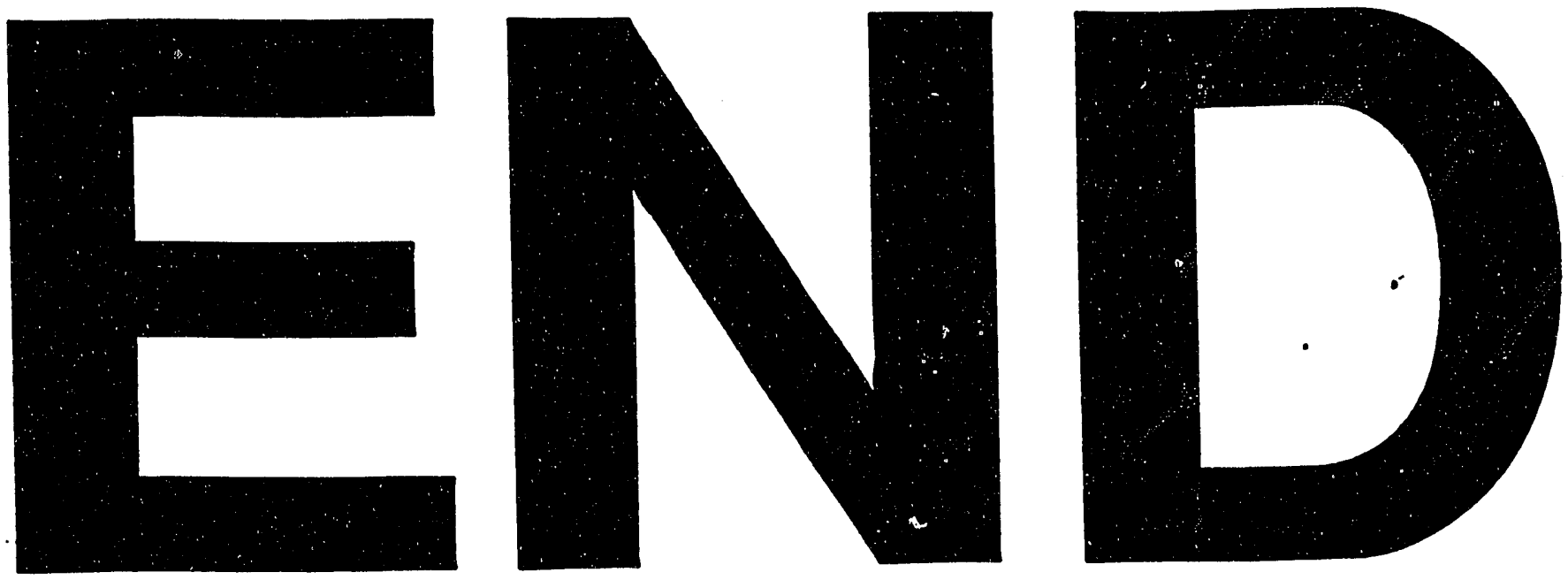
